\title{
The FRED Flow-Diverter Stent for Intracranial Aneurysms: Clinical Study to Assess Safety and Efficacy
}

\author{
M.A. Möhlenbruch, C. Herweh, L. Jestaedt, S. Stampfl, S. Schönenberger, P.A. Ringleb, M. Bendszus, and M. Pham
}

\begin{abstract}
BACKGROUND AND PURPOSE: Flow-diverter stents are emerging for the endovascular treatment of difficult-to-treat or otherwise untreatable cerebral aneurysms (wide-neck, fusiform, dissecting, blisterlike, or giant). We assessed the clinical safety and efficacy of the Flow-Redirection Endoluminal Device.

MATERIALS AND METHODS: This was an institutional review board-approved single-center observational clinical study in 29 patients with 34 aneurysms elected to be treated by endovascular intervention. After providing informed consent, patients were included according to the following criteria: aneurysm fundus-to-neck ratio $<2$ or neck diameter $>4 \mathrm{~mm}$, fusiform, dissecting, or giant aneurysms. The primary end point for clinical safety was the absence of death, absence of major or minor stroke, and absence of transient ischemic attack. The primary end point for treatment efficacy was complete angiographic occlusion according to the O'Kelly Marotta grading scale immediately after the procedure and at follow-up after 3 and 6 months (O'Kelly Marotta D: complete occlusion).

RESULTS: The Flow-Redirection Intraluminal Device deployment was technically successful in all cases. In 26/29 (89\%) of patients, the primary end point of safety was reached; in the 3 remaining patients, 1 disabling ischemic stroke and 2 minor strokes with complete recovery at follow-up were observed. Angiographic (DSA and MRA) and clinical follow-up were available after 3 months in 29/29 (100\%) and after 6 months in 25/29 (86\%) patients (after 6 months, only MRA follow-up was performed according to our study protocol and institutional standard). At 3-month follow-up, complete occlusion was reached in 19/34 aneurysms (O'Kelly Marotta D: 19/34; 56\%). At 6-month follow-up, aneurysm occlusion was complete in 22/30 aneurysms (O'Kelly Marotta D: 22/30; 73\%).
\end{abstract}

CONCLUSIONS: Deployment of the Flow-Redirection Intraluminal Device flow-diverter stent is safe and effective in the treatment of difficult-to-treat or otherwise untreatable intracranial aneurysms.

ABBREVIATIONS: FD = flow-diverter; FRED = Flow-Redirection Intraluminal Device; LTA = light transmission aggregometry; OKM = O'Kelly Marotta grading scale; PTA $=$ percutaneous transluminal angioplasty

$E$

ndovascular treatment of intracranial aneurysms by coiling has become an accepted alternative to surgical clipping, with increasing evidence for lower morbidity and mortality rates, especially in clinical equipoise. ${ }^{1-3}$ However, especially in wide-neck, fusiform, dissecting, and giant aneurysms, incomplete coiling and reperfusion are still a major limitation preventing stable long-term occlusion. Aneurysm recanalization and/or neck remnants may be observed despite

Received August 7, 2014; accepted after revision December 3.

From the Departments of Neuroradiology (M.A.M., C.H., L.J., S. Stampfl, M.B., M.P.) and Neurology (S. Schönenberger, P.A.R.); University of Heidelberg Medical Center, Heidelberg, Germany.

Please address correspondence to Markus A. Möhlenbruch, MD, Department of Neuroradiology, University of Heidelberg Medical Center, Im Neuenheimer Feld 400, 69120 Heidelberg, Germany; e-mail: markus.moehlenbruch@med.uni-

heidelberg.de

= Indicates article with supplemental on-line tables.

http://dx.doi.org/10.3174/ajnr.A4251 further refinement in coil technology such as coated platinum coils ${ }^{4}$ and/or procedural modification such as the balloon-remodeling technique ${ }^{5}$ or stent-assisted coil embolization. ${ }^{6}$

The development of flow-diverter (FD) stents has offered the potential of aneurysm occlusion through thrombosis triggered by the disruption of flow into the aneurysm sac. ${ }^{7-16}$ As a key element of construction, these stents have a braided mesh with a densely covered surface. Once the FD is expanded to cover the aneurysm neck, thrombosis is induced by stasis of flow within the aneurysmal sac. The porosity of the FD mesh and the pressure gradient between parent and smaller adjacent branch vessels preserve flow and patency of the latter even if covered. The Flow-Redirection Endoluminal Device (FRED; MicroVention, Tustin, California) is a new generation of FDs for reconstruction of the parent artery and aneurysm occlusion. Its unique dual-layer design composed of a low-porosity inner mesh and a high-porosity outer stent may provide potential advantages over other available FDs in safe deliverability and effective occlusion 
of the target lesion. We report our analysis of the clinical safety and efficacy of the FRED in 29 patients with 34 aneurysms.

\section{MATERIALS AND METHODS \\ Patient Selection}

Approval for prospective data collection of all interventional procedures reported in this study was given by the institutional review board of the medical faculty. Informed consent for study inclusion was obtained from the patients or their legal representatives. Patients were enrolled between February 2013 and July 2014.

\section{Criteria of Inclusion/Exclusion}

Patients with intracranial aneurysms were included in the study and treated with the FRED if the aneurysms satisfied the following morphologic and morphometric criteria of inclusion: 1) wideneck saccular (fundus-to-neck ratio $<2$ or neck diameter $>4$ $\mathrm{mm}$ ), 2) fusiform or circumferential, 3) dissecting, 4) blisterlike (broad-based at a typical location, eg, the supraclinoid side wall of the ICA and $\leq 2 \mathrm{~mm}$ ), and 5) giant (defined as $\geq 25-\mathrm{mm}$ maximum diameter) or those presenting with mass effect. We considered these aneurysms to have a high likelihood of technical failure and/or recurrence with conventional endovascular techniques. Five patients $(5 / 29,17 \%)$ with recanalization after previous coiling were enrolled. Exclusion criteria were consensus about clipping as the appropriate treatment option in cases with unfavorable branch configuration with an acute angle in relation to the parent vessel or an unfavorably small diameter of the parent artery of $\leq 2.0 \mathrm{~mm}$.

\section{FRED}

The FRED is an FD designed for the treatment of intracranial aneurysms. The device consists of a braided self-expandable closed-cell dual-layer stent (also referred to a "stent within a stent") with a low-porosity inner mesh of higher pore attenuation (48 nitinol wires) and an outer stent with high porosity (16 nitinol wires). This dual-layer design is restricted to the midsection of the stent and encompasses $80 \%$ of its total length. An interwoven double helix of radiopaque tantalum strands attaches the inner mesh to the outer stent and improves visibility over its full length of dual-layer coverage. Each end of the stent is flared and marked by 4 radiopaque tips. Currently, the FRED is available in 5 different diameters $(3.5,4.0,4.5,5.0$, and $5.5 \mathrm{~mm}$ ) recommended for vessel diameters from 2.5 to $5.5 \mathrm{~mm}$ and at working lengths (duallayer coverage) from 7 to $56 \mathrm{~mm}$. The FRED is attached to a delivery microwire with a radiopaque distal tip and a proximal marker. If the device is not correctly positioned across the aneurysm neck, the pusher allows resheathing and repositioning of the stent as long as $\leq 80 \%$ of its length has been unsheathed/deployed. As soon as the 0.027-inch delivery-microcatheter (Headway 27; MicroVention) is fully withdrawn over the delivery microwire, the coupling wire releases and the stent is deployed.

\section{Description of Technique}

Every procedure was performed via a transfemoral approach with the patient under general anesthesia. A $6 \mathrm{~F}-8 \mathrm{~F}$ guiding catheter was introduced through a femoral sheath into the carotid or ver- tebral artery. The radiologic examination of the target vessel was performed by using a biplane angiographic system (Artis zee biplane; Siemens, Erlangen, Germany) and 3D rotational angiography. FD length was chosen according to the length of the aneurysm neck with the procedural goal to ensure arterial wall coverage with the inner mesh extending to at least $2 \mathrm{~mm}$ beyond both the distal and proximal limits of the neck. For FD delivery, a Headway 27 microcatheter was navigated past the aneurysm neck with a microguidewire, Traxcess (MicroVention) or Radiofocus Guide Wire GT 16 (Terumo, Tokyo, Japan). Under roadmap guidance, the FD was unsheathed by slowly withdrawing the delivery microcatheter for 2-3 minutes while the delivery wire was gently pushed on to facilitate complete opening and wall apposition of the FD. An in-stent percutaneous transluminal angioplasty (PTA) was performed with a balloon microcatheter (HyperGlide or HyperForm; Covidien, Irvine, California) if incomplete opening was observed on $2 \mathrm{D}$ angiography or $3 \mathrm{D}$ DynaCT (Siemens). In those cases in which additional aneurysm coiling was performed, a microcatheter was initially positioned inside the aneurysmal sac followed by jailing of the microcatheter between the parent vessel wall and the FD at deployment.

\section{Anticoagulation/Antiplatelet Regimen}

Patients were prepared with aspirin, $100 \mathrm{mg}$, and clopidogrel, 75 $\mathrm{mg}$ (loading dose of $300 \mathrm{mg}$ ), 5 days before treatment. During the procedure, anticoagulation was initiated with a bolus of standard heparin $(70-100 \mathrm{IU} / \mathrm{kg})$ followed by intravenous administration to maintain an activated clotting time of $\geq 250$ seconds. After the procedure, a daily dose of clopidogrel $(75 \mathrm{mg})$ and aspirin (100 $\mathrm{mg}$ ) was given for 3 and 6 months, respectively. Platelet inhibition was tested by using the light transmission aggregometry (LTA) method on the day before or immediately before starting the procedure. If according to this test, the patient was considered a nonresponder (LTA result of $<6 \Omega$ ), an LTA test with 2-MeS-AMP (selective P2Y12-adenosine diphosphate receptor antagonist) was performed. In case of an LTA result with 2 -MeS-AMP $\leq 5 \Omega$, a pharmacokinetic nonresponse was suspected and a reloading dose of clopidogrel (300 mg) was administered. In the case of repeated test failure even after reloading or an LTA result with $2-\mathrm{MeS}-\mathrm{AMP}>5 \Omega$, pharmacodynamic resistance was assumed and prasugrel was substituted for clopidogrel. In patients with ruptured aneurysms, tirofiban was administrated intravenously during the endovascular procedure before FD deployment and aspirin and clopidogrel were started after the procedure.

\section{Evaluation of End Points}

Safety. Clinical evaluation by detailed neurologic examination was performed immediately after the procedure, at 2 hours after the procedure, and during the clinical visit on the following day and was finally scored by the mRS at discharge and follow-up by a board-certified neurologist not blinded to treatment. Technical safety, including navigation, visibility, deployment, opening, and vessel wall apposition of the FD, were rated by 2 senior neurointerventionalists (M.A.M., M.P.).

Efficacy. Each angiographic result was categorized according to the O'Kelly Marotta (OKM) grading scale for assessment of cere- 
bral aneurysms treated by flow diversion. ${ }^{17}$ Each aneurysm was assigned an occlusion grade according to the initial degree of filling $(\mathrm{A}=$ total filling, $\mathrm{B}=$ subtotal filling, $\mathrm{C}=$ entry remnant, $\mathrm{D}=$ no filling) and the degree of stasis (prolongation of stasis into $1=$ arterial, 2 = capillary, 3 = venous phase). Detailed follow-up examinations were performed with both DSA and MR imaging (3T, Magnetom Trio or Magnetom Verio; Siemens; TOF MRA with and without contrast agent) at 3 months. After 6 months, MRA was exclusively performed. To evaluate thrombosis of the aneurysm and shrinkage of the aneurysm sac, we measured the maximum diameters of all lesions on preprocedural MR images in the axial plane with 2 additional orthogonal planes and compared them with MR imaging at 3 and 6 months. Aneurysm morphometry on MR imaging was categorized into 3 groups: 1) complete or partial ( $\geq 20 \%$ ) decrease, 2) stable, 3) progressive ( $\geq 10 \%$ ).

\section{RESULTS}

Twenty-nine patients ( 14 female, 15 male; mean age, 58 years; age range, 32-80 years) with 34 aneurysms fulfilled the criteria of inclusion. Patient demographics and aneurysm features are shown in On-line Table 1, and clinical and angiographic details are listed in On-line Table 2.

After preparation with aspirin and clopidogrel, a pharmacokinetic resistance was suspected in 1 patient. Following a reloading dose of clopidogrel, responder status was confirmed. In another patient, pharmacodynamic resistance was assumed on the basis of LTA results and prasugrel was substituted for clopidogrel. Twenty-three aneurysms originated from the ICA/posterior communicating artery; 3 , from the anterior cerebral artery; and 8 were located in the posterior circulation. We included 17 wide-neck saccular aneurysms, 3 fusiform/dissecting aneurysms, 4 blisterlike aneurysms, and 10 large/giant aneurysms (including 2 partially thrombosed aneurysms). Nine aneurysms were symptomatic, and 5 aneurysms were in acute/subacute stages of SAH. In all patients and for all aneurysms, the FRED could be navigated to the target area and was deployed successfully across the aneurysm neck with complete neck coverage.

Thirty-two of 34 (94\%) aneurysms were treated by deployment of a single FD. Two patients had undergone previous endovascular treatment with stents for the same target lesion: One patient with reperfusion was previously treated by stent-assisted coiling (LEO + stent; Balt Extrusion, Montmorency, France); the other patient with a 16.3 -mm large-neck cavernous ICA aneurysm was pretreated with 2 overlapping LEO + stents $(3.5 \times 50$ $\mathrm{mm}$ ). Median diameters of the stented parent vessel segments were proximally $3.8 \mathrm{~mm}$ (range, $2.0-5.3 \mathrm{~mm}$ ) and distally $3.4 \mathrm{~mm}$ (range, 1.9-5.7 mm). In 10 cases, bioactive coils (Cerecyte; Codman Neurovascular, Raynham, Massachusetts) were used with a median packing attenuation of $20 \%$ (range, 9\%-43\%). A PTA before FD deployment was performed in 1 patient with a $50 \%$ stenosis directly proximal to a fusiform vertebral aneurysm. PTA after FD deployment was performed in 5 patients with paraophthalmic or supraclinoid ICA aneurysms. In all of these 5 patients, near-complete $(\geq 90 \%)$ wall apposition of the FD had been achieved before PTA; however, PTA was elected to further improve wall apposition and opening of the vessel diameter. At termination of the procedure, an immediate change of angiographic filling (OKM grading scale) was achieved in 33/34 (97\%) aneurysms with complete or near-complete early occlusion (OKM C or D) occurring in $9 / 34(26 \%)$ within 30 minutes after FD deployment.

\section{Clinical Safety and Procedure-Related Complications}

The primary end point of safety (absence of any new transient or permanent neurologic deficit or death) was reached in 26/29 (90\%) patients. A new permanent neurologic deficit by major stroke occurred in 1 of the treated patients after the procedure. Minor strokes were observed in 2 patients ( 1 immediately postprocedural and 1 after 2 months [see below]) who recovered completely and were each asymptomatic after 3 months of follow-up $(\mathrm{NIHSS}=0, \mathrm{mRS}=0)$. Neither mortality nor transient ischemic symptoms occurred in any of the treated patients after the procedure and throughout follow-up.

The major stroke occurred in a patient with a ruptured aneurysm of a short paramedian circumferential perforator branch emerging from the basilar trunk. This very small aneurysm measured $1.6 \mathrm{~mm}$ and arose from the midsection of the basilar trunk just distal to the AICA offspring. It was unmasked only on the repeat angiogram 12 days after $\mathrm{SAH}$ and was not visible on the initial angiogram obtained on the day of SAH. Weight-adapted tirofiban was started intravenously with heparin after femoral access and was maintained for 12 hours. The shortest available FRED $(3.5 \times 7 \mathrm{~mm})$ was successfully deployed to cover the midsection of the basilar trunk, and the control angiogram demonstrated immediate contrast stasis within the dome with preserved flow through the perforator. Nine hours after FRED deployment, the patient developed a right-sided high-grade hemiparesis and dysarthria. DWI MR imaging was performed immediately and confirmed a left paramedian pontine ischemic infarction at the level of the aneurysm. At discharge, the patient had a Glasgow Coma Scale score of 15 with substantially improved motor strength on her right side.

One minor stroke occurred in a patient with a superior cerebellar artery aneurysm 1 day after FRED deployment. DWI MR imaging confirmed a few microembolic infarcts in the ipsilateral superior cerebellar artery territory. Immediate control DSA showed intact duplicated superior cerebellar arteries and contrast stasis within the dome without any evidence of local thrombus formation inside the FD or thromboembolic branch occlusion distal to it. The patient was discharged home with a residual mild unsteadiness of gait, which had completely resolved at 3-month follow-up.

The second above-mentioned minor stroke occurred in a patient with a giant partially thrombosed ICA aneurysm 2 months after FD deployment. Because of infection of his ventriculoperitoneal shunt, which required conversion to a ventriculoatrial shunt, prasugrel (the patient was a nonresponder to aspirin and clopidogrel) was terminated. After 1 week of anticoagulation on heparin only, the patient developed sudden hemiparesis due to complete FD in-stent thrombosis with complete ICA occlusion confirmed by Doppler sonography and emergency DSA. Resolution of symptoms could be observed clinically during emergency DSA in the awake state after pharmacologic induction of controlled hypertension with DSA documentation of sufficient col- 
lateral circulation through the circle of Willis and leptomeningeal anastomoses. MR imaging the next day showed development of only microembolic watershed infarcts and absence of any cortical or basal territorial infarction. Full resolution of symptoms was observed in this patient at discharge, and stable ICA occlusion without any evidence of residual antegrade flow was documented by Doppler sonography and MR imaging. None of the other patients developed any new transient or permanent neurologic deficits during follow-up.

An asymptomatic in-stent thrombosis in the acute stage after SAH Hunt and Hess $3^{\circ}$ was observed in 1 other patient with a dissecting aneurysm of AICA origin. Appositional clot formation occurred within the FD at the level of the aneurysm neck 5 minutes after FD deployment. This patient had received prior weightadapted tirofiban (30 minutes before deployment) and heparin, and the clot dissolved after pharmacologic induction of controlled hypertension. Subsequently, DWI MR imaging ruled out brain stem infarction, and the patient did not develop any new symptoms.

\section{Routine Follow-Up (Clinical, DSA, MRA)}

Complete angiographic (DSA and MRA) and clinical follow-up could be performed after 3 months in 29/29 (100\%) and after 6 months in 25/29 (86\%) patients (after 6 months only MRA was performed according to our study protocol and institutional standard). At 3-month follow-up, a complete occlusion (OKM D) was achieved in 19/34 (56\%) aneurysms. A near-complete occlusion (OKM C) was reached in 12/34 (35\%) aneurysms. Reduction of aneurysmal sac filling and prolongation of stasis were noted in $27 / 34(79 \%)$ aneurysms from the initial postprocedural control DSA to the 3-month follow-up control DSA. At 6-month followup, 7/30 (23\%) aneurysms showed a decrease of residual inflow, and none developed recurrence with enlargement of the aneurysm at the base. At 6-month follow-up, aneurysm occlusion was complete (OKM D) in 22/30 (73\%) aneurysms. A near-complete occlusion (OKM C) was detected in another $8 / 30$ (27\%) aneurysms. On follow-up angiograms, in no case was any in-stent stenosis, in-stent thrombosis, or migration of the FRED implant observed.

MR imaging analysis at 3 months showed shrinkage or complete disappearance of the thrombosed aneurysm in 59\% (20/34 aneurysms) and no change in size in 41\% (14/34 aneurysms including 10 additional aneurysm coilings). On MR imaging at 6 months, shrinkage or complete disappearance was observed in $70 \%$ (21/30 aneurysms) with no change in 30\% (9/30 of aneurysms, including 8 that were additionally coiled).

Two illustrative cases are demonstrated in Figs 1 and 2.

\section{DISCUSSION}

In this single-center prospective clinical study, the FRED was evaluated for the treatment of otherwise untreatable or difficult-totreat intracranial aneurysms (ie, wide-neck, fusiform, dissecting, blisterlike, or giant). This new generation of intracranial FD appeared to be a promising safe and effective alternative treatment to other FDs for these complex aneurysms. The primary end point for clinical safety (ie, absence of death and major or minor stroke

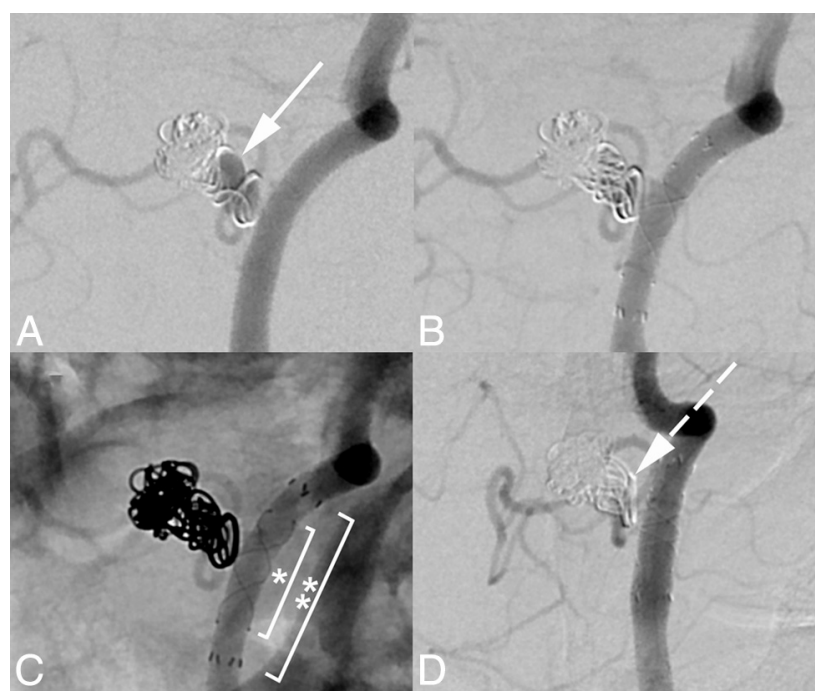

FIG 1. Patient 1 with a 4.4-mm residual aneurysm of the initial posterior inferior cerebellar artery segment close to its origin from the V4 segment of the left vertebral artery (solid white arrow, A). After recoiling, we implanted a FRED $3.5 \times 13 \mathrm{~mm}$ to cover the orifice of the posterior inferior cerebellar artery origin $(B)$. The flow-diverting working zone (inner stent) is centrally positioned over the posterior inferior cerebellar artery origin (solid white bracket marked with 1 asterisk, C). The outer stent, which provides stable vessel anchorage symmetrically, overlaps the proximal and distal edge of the inner flow-diverting stent. Its distal flared ends are marked with solid white brackets and 2 asterisks (C). At 3-month follow-up, complete occlusion of the residual aneurysm was observed, with no residual inflow $(D)$, which was also verified on different projections and 3D DSA. At the base of the coil basket, the overprojection of a proximal posterior inferior cerebellar artery loop (dashed white arrow, D) simulates residual aneurysm filling, but contrast washes out rapidly at this position after the arterial phase (not shown). Complete occlusion was also confirmed by unenhanced and enhanced TOF-MRA at 3 and 6 months.

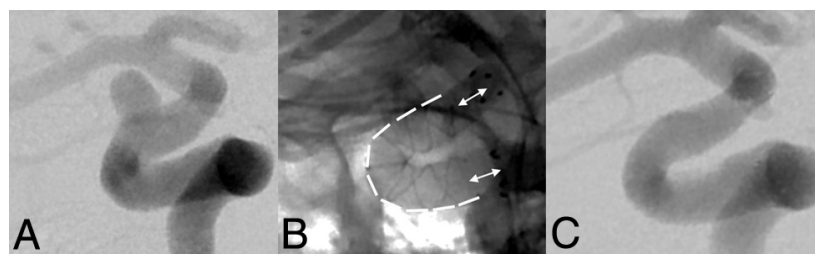

FIG 2. Patient 7 with a $6.5-\mathrm{mm}$ supraclinoid ICA aneurysm (A). In this case, a FRED $4.5 \times 25 \mathrm{~mm}$ could be placed to cover the aneurysm neck with the inner stent mesh having the flow-diverting effect (dashed white line). Complete wall apposition can be appreciated along the entire carotid siphon $(B)$. The outer stent ensuring stable vessel wall anchorage overlaps the inner flow-diverting stent over $3.5 \mathrm{~mm}$ at its proximal and distal ends (double-headed white arrows, B). At its termination, 4 radiopaque tips mark the flared ends. At 3-month followup, complete occlusion of the aneurysm could be confirmed on DSA (C) with no residual inflow, which was also confirmed by MR imaging at that time and remained stable at 6-month follow-up.

and absence of transient ischemic attack) was reached in 26/29 (90\%) treated patients.

The observed overall permanent morbidity of 3.4\% and mortality of $0 \%$ in our study was low. In other prospective clinical studies on different intracranial FDs (Pipeline Embolization Device; Covidien, Irvine, California; Silk+, Balt Extrusion; Surpass stent; Stryker Neurovascular, Fremont, California), similar end points occurred with $0 \%-15 \%$ permanent neurologic deficits and $0 \%-8 \%$ mortality. ${ }^{7-16}$ 
Altogether, we report in this study that the end point of clinical safety was not achieved in 2 patients with minor ischemic stroke, in whom complete resolution of symptoms was observed after 3-month follow-up and in 1 patient with major ischemic stroke. In the latter patient, the only one with a permanent neurologic deficit, an exceptionally rare and difficult-to-treat aneurysm was targeted. Only repeat DSA but not initial DSA revealed a ruptured aneurysm of a perforator branch of the trunk of the basilar artery. In the literature, only 13 cases were reported with this type of aneurysm. ${ }^{18,19}$ Because this aneurysm was not amenable to any coiling or neck-clipping technique, we decided in favor of treatment with flow diversion by using the FRED. Despite sufficiently effective antiplatelet therapy according to platelet function tests, however, the patient's symptoms, consistent with a delayed perforator stroke 9 hours after FRED deployment, were confirmed by DWI. The exact location and anatomic extension of the ischemic pontine DWI lesion was consistent with delayed occlusion of the perforator branch harboring the aneurysm and was likely the inevitable effect of decreased flow in this vessel. ${ }^{20}$

In one patient who had microembolic infarcts in the superior cerebellar artery territory, from which she recovered completely at 3-month follow-up, a similar flow-related pathogenesis seems most plausible. In a larger series, only observing 32 posterior circulation aneurysms treated with FDs, Phillips et al ${ }^{12}$ reported a permanent neurologic complication rate of $9.4 \%$, with perforator territory infarctions occurring in 14\% of the subgroup of patients with basilar artery aneurysms. These authors concluded that treatment with an FD is safe and effective but should be reserved for cases not easily or effectively treatable with conventional endovascular techniques, owing to the high rate of ischemic perforator infarcts following FD use in the posterior circulation and the basilar artery trunk in particular. ${ }^{12}$

Two cases (2/29 patients, $6.9 \%)$ of in-stent thrombosis occurred in our study: one appositional clot formation in the acute stage of SAH, which was fully reversible on angiography and without clinical consequences under controlled hypertension together with weight-adapted tirofiban and heparin; and the other at 2 months after termination of prasugrel because of infection of his ventriculoperitoneal shunt requiring surgical intervention for conversion into a ventriculoatrial shunt. No further delayed thromboembolic events after the procedure were detected in any other patient by occurrence of clinical symptoms or by follow-up MR imaging.

In the present trial, FD deployment was technically successful in all cases. Insufficient opening of the FD, which is reported for other available FDs to occur in up to $10 \%$ of cases, was not observed in any case for the FRED in our study. ${ }^{8,9,16}$ We elected to perform in-stent PTA of the FRED if the slightest suspicion of incomplete wall apposition arose on the immediate postdeployment control angiograms (5/29 patients, $17 \%)$. This operation standard was chosen because we consider the risk of thrombus formation and subsequent parent artery occlusion to be high if suboptimal wall apposition is tolerated. ${ }^{11,15}$ In those 5 cases in which we performed PTA within the FD stent (all were paraophthalmic or supraclinoid ICA aneurysms with sharp angulation of the carotid siphon), opening of the stent before PTA was still near-complete with at least $90 \%$ of the stent diameter and was further improved to $100 \%$ after PTA. To ensure proper opening and full deployment of the FRED, we ensured that unsheathing and release of the FRED occurred in a slow and controlled fashion during several minutes by gentle application of the push/pull technique with the delivery microcatheter tip properly held in a central position within the central vessel lumen. However, the operator should be aware that even if these precautions are taken, incomplete opening still may occur, which should prompt complete retrieval of the device by simply resheathing it into the delivery microcatheter.

On follow-up DSA at 3 months, no in-stent stenosis or changes in stent morphology such as "fish mouth" (ie, inward crimping of 1 or both ends of FD) or "foreshortening" phenomena were observed, which were described in an early series by Kocer et $\mathrm{al}^{21}$ in 5 cases with a FRED. In our series of 29 patients, we exclusively used the second-generation version of the FRED, which obtained approval for human clinical use within the European community by CE marking in December 2012. Evaluation of the efficacy of occlusion showed promising results for the FRED in otherwise untreatable or difficult-to-treat aneurysms during early follow-up until 3 and 6 months postoperatively. Diagnostic follow-up was achieved not only by invasive angiographic but also MR angiographic evaluation, including contrast-enhanced TOF-MR angiography considered to be sensitive for residual aneurysmal inflow, especially when nonenhanced TOF-MR angiography source images are used for subtraction. At 3-month followup, complete occlusion (OKM D) was achieved in 19/34 (56\%) aneurysms. At 6-month follow-up, aneurysm occlusion was complete in 22/30 (73\%) aneurysms defined as an OKM grade of " $D$ " (complete occlusion), which, on the Raymond and Roy scale, would correspond to grade $1^{\circ} .^{22}$

Compared with other prospective clinical studies on different intracranial FDs (the Pipeline Embolization Device, SILK+, and Surpass), our efficacy rate of $86 \%$ in aneurysms of $<10 \mathrm{~mm}$ was similar to the reported complete occlusion rate at 6 months of $49 \%-93 \%{ }^{7-16,23}$ However, in aneurysms of $>10 \mathrm{~mm}$, our occlusion rates were lower than the rates reported in the Pipeline Embolization Device for Uncoilable or Failed Aneurysms trial ${ }^{15}$ and in the recent meta-analysis from Brinjikji et al. ${ }^{23}$ One possible explanation is that the low number of aneurysms of $>10 \mathrm{~mm}$ in our study $(n=11)$ might have led to deviations from the population mean, which, due to the larger group size, might have been estimated more accurately in a larger study such as the Pipeline Embolization Device for Uncoilable or Failed Aneurysms trial, for example. Another possible explanation is that we required the 6-month follow-up to include TOF-MRA and applied rigorous aneurysm evaluation of nonenhanced TOF, contrast-enhanced TOF and, importantly, also on subtracted TOF images on which we rated any suspicion of residual inflow or very slow inflow as noncomplete occlusion.

In experimental aneurysm models and also in human studies, it could be regularly observed that FD stents can induce complete and stable occlusion of the aneurysm sac by stasis of flow and subsequent thrombosis, even without additional introduction of coils. In 10 patients, aneurysm occlusion was observed after adjunctive use of coils, introduced by a jailed microcatheter during the same treatment session before FD deployment. In our current 
practice, we use adjunctive coiling if aneurysms are large or giant, in the acute stage of SAH, or in cases of retreatment/recurrence of previously ruptured aneurysms pretreated by coiling. The primary rationale for adjunctive coiling in our view is to prevent aneurysm rupture and to enhance stable long-term occlusion. ${ }^{24}$ Additional coiling may not only prevent early or delayed rupture but also, especially for extremely wide-neck aneurysms, provide good mechanical support and stabilization for FD deployment and a stable long-term position, in that (partial) herniation or bulging of the FD into the aneurysm sac can be avoided.

The recently introduced FRED may offer several potential advantages over other currently available intracranial FDs: 1) Due to its dual-layer design and availability in long sizes of up to $56 \mathrm{~mm}$, the scaffolding effect of the stent for obtaining outward stability toward the wall of the parent vessel seems to be enhanced and, most important, is achievable in a single treatment session, which makes this device especially useful in fusiform or giant aneurysms, whereas 2 sequential procedures might have been necessary before (scaffolding stent first followed by FD implantation). 2) The outer stent is woven with 16 wires leading to lower friction within the delivery microcatheter, thus facilitating deployment especially of longer stents — other available FDs, for example, are composed of 48 wires (Silk + and Pipeline Embolization Device) or 96 wires (Surpass). 3) The additive radial force vectors of the inner and outer stent provide a high degree of reliability of stent opening, a feature especially critical when deployment includes ICA segments around the carotid siphon. 4) There is improved lamination of blood flow through its dual-layer design and higher pore attenuation with $16+48$ wires compared with 48 wires of the Pipeline Embolization Device and Silk. ${ }^{16,25,26}$ 5) There is lower vessel wall coverage in the longitudinal direction because of the shorter inner stent (shortest available on the market), which is designed to limit the working layer mainly to the neck of the aneurysm and to spare adjacent branches or perforator arteries, to maintain the patency of these vessels. 6) There is enhanced visibility of the central stent segment and of its distal and proximal ends.

We acknowledge that the major limitations of this prospective study include the limited number of 29 patients, a short follow-up period, MRA only after 6 months, and lack of randomized comparisons with other potentially efficacious therapies. Furthermore, most aneurysms were located at the paraophthalmic segment of the ICA, the mean aneurysm size was relatively small, and not all cases were treated solely by FDs but additional coiling was performed in some cases. However, recruitment followed clearly defined criteria of inclusion/exclusion, and clinical/radiologic follow-up was rigorous, including not only invasive angiography but also MR imaging to measure accurately the shrinkage of the aneurysm sac and also nonenhanced and contrast-enhanced TOF-MR angiography to detect even small amounts of slow residual inflow that may be silent on invasive angiography. Furthermore, our cohort is the largest so far in which the safety and efficacy profile of this new generation of intracranial FDs has been investigated and may serve as a basis for subsequent larger and multicenter studies.

\section{CONCLUSIONS}

The FRED for treatment of difficult-to-treat or otherwise untreatable intracranial aneurysms was prospectively observed in this clinical study to provide a high degree of safety and efficacy, measured as complete aneurysm occlusion on DSA and MR imaging during 6-month follow-up. Long-term durability and safety still remain to be proved by larger series and after prolonged followup. We report that it is reasonable, safe, and effective to use intracranial FDs such as the FRED, especially for targeting otherwise untreatable or difficult-to-treat complex intracranial aneurysms.

Disclosures: Christian Herweh—UNRELATED: Travel/Accommodations/Meeting Expenses Unrelated to Activities Listed: MicroVention, Comments: Brain AVM Congress 2014 (Nancy, France), travel expenses and registration fee. Peter A. Ringleb-UNRELATED: Consultancy: Boehringer Ingelheim, Bayer; Grants/Grants Pending: German Research Foundation; Payment for Lectures (including service on Speakers Bureaus): Boehring Ingelheim, Bayer, Bristol-Myers Squibb, Pfizer, PAION; Payment for Development of Educational Presentations: Boehringer Ingelheim; Travel/Accommodations/Meeting Expenses Unrelated to Activities Listed: Bayer. Martin Bendszus_UNRELATED: Board Membership: data and safety monitoring board Vascular Dynamics; Consultancy: Codman, Roche, Guerbet; Payment for Lectures (including service on Speakers Bureaus): Roche, Novartis, Guerbet, Codman. Mirko Pham-UNRELATED: Other: Memorial Stipend from Else-Kröner-Fresenius-Foundation.

\section{REFERENCES}

1. Alshekhlee A, Mehta S, Edgell RC, et al. Hospital mortality and complications of electively clipped or coiled unruptured intracranial aneurysm. Stroke 2010;41:1471-76

2. Brinjikji W, Rabinstein AA, Nasr DM, et al. Better outcomes with treatment by coiling relative to clipping of unruptured intracranial aneurysms in the United States, 2001-2008. AJNR Am J Neuroradiol 2011;32:1071-75

3. Molyneux A, Kerr R, Stratton I, et al. International Subarachnoid Aneurysm Trial (ISAT) of neurosurgical clipping versus endovascular coiling in 2143 patients with ruptured intracranial aneurysms: a randomised trial. Lancet 2002;360:1267-74

4. White PM, Lewis SC, Gholkar A, et al. Hydrogel-coated coils versus bare platinum coils for the endovascular treatment of intracranial aneurysms (HELPS): a randomised controlled trial. Lancet 2011;377:1655-62

5. Moret J, Cognard C, Weill A, et al. The "Remodelling Technique" in the treatment of wide neck intracranial aneurysms: angiographic results and clinical follow-up in 56 cases. Interv Neuroradiol 1997;3:21-35

6. Shapiro M, Becske T, Sahlein D, et al. Stent-supported aneurysm coiling: a literature survey of treatment and follow-up. AJNR Am J Neuroradiol 2012;33:159-63

7. Lylyk P, Miranda C, Ceratto R, et al. Curative endovascular reconstruction of cerebral aneurysms with the Pipeline embolization device: the Buenos Aires experience. Neurosurgery 2009;64:632-42; discussion 642-43; quiz N6

8. Lubicz B, Collignon L, Raphaeli G, et al. Flow-diverter stent for the endovascular treatment of intracranial aneurysms: a prospective study in 29 patients with 34 aneurysms. Stroke 2010;41:2247-53

9. Byrne JV, Beltechi R, Yarnold JA, et al. Early experience in the treatment of intra-cranial aneurysms by endovascular flow diversion: a multicentre prospective study. PLoS One 2010;5. pii: e12492

10. Nelson PK, Lylyk P, Szikora I, et al. The Pipeline embolization device for the intracranial treatment of aneurysms trial. AJNR Am J Neuroradiol 2011;32:34-40

11. McAuliffe $\mathrm{W}$, Wycoco $\mathrm{V}$, Rice $\mathrm{H}$, et al. Immediate and midterm results following treatment of unruptured intracranial aneurysms with the Pipeline embolization device. AJNR Am J Neuroradiol 2012;33:164-70

12. Phillips TJ, Wenderoth JD, Phatouros CC, et al. Safety of the Pipeline 
embolization device in treatment of posterior circulation aneurysms. AJNR Am J Neuroradiol 2012;33:1225-31

13. Pistocchi S, Blanc R, Bartolini B, et al. Flow diverters at and beyond the level of the circle of Willis for the treatment of intracranial aneurysms. Stroke 2012;43:1032-38

14. Yu SC, Kwok CK, Cheng PW, et al. Intracranial aneurysms: midterm outcome of Pipeline embolization device: a prospective study in 143 patients with 178 aneurysms. Radiology 2012;265:893-901

15. Becske T, Kallmes DF, Saatci I, et al. Pipeline for uncoilable or failed aneurysms: results from a multicenter clinical trial. Radiology 2013;267:858-68

16. De Vries J, Boogaarts J, Van Norden A, et al. New generation of flow diverter (Surpass) for unruptured intracranial aneurysms: a prospective single-center study in 37 patients. Stroke 2013;44:1567-77

17. O'Kelly CJ, Krings T, Fiorella D, et al. A novel grading scale for the angiographic assessment of intracranial aneurysms treated using flow diverting stents. Interv Neuroradiol 2010;16:133-37

18. Gross BA, Puri AS, Du R. Basilar trunk perforator artery aneurysms: case report and literature review. Neurosurg Rev 2013;36:163-68

19. Chalouhi N, Jabbour P, Starke RM, et al. Treatment of a basilar trunk perforator aneurysm with the Pipeline embolization device: case report. Neurosurgery 2014;74:E697-701
20. Roszelle BN, Babiker MH, Hafner W, et al. In vitro and in silico study of intracranial stent treatments for cerebral aneurysms: effects on perforating vessel flows. J Neurointerv Surg 2013;5:354-60

21. Kocer N, Islak C, Kizilkilic O, et al. Flow Re-direction Endoluminal Device in treatment of cerebral aneurysms: initial experience with short-term follow-up results. J Neurosurg 2014;120:1158-71

22. Raymond J, Guilbert F, Weill A, et al. Long-term angiographic recurrences after selective endovascular treatment of aneurysms with detachable coils. Stroke 2003;34:1398-403

23. Brinjikji W, Murad MH, Lanzino G, et al. Endovascular treatment of intracranial aneurysms with flow diverters: a meta-analysis. Stroke 2013;44:442-47

24. Kulcsár Z, Houdart E, Bonafé A, et al. Intra-aneurysmal thrombosis as a possible cause of delayed aneurysm rupture after flow-diversion treatment. AJNR Am J Neuroradiol 2011;32:20-25

25. Kim M, Levy EI, Meng H, et al. Quantification of hemodynamic changes induced by virtual placement of multiple stents across a wide-necked basilar trunk aneurysm. Neurosurgery 2007;61:130512; discussion 1312-13

26. Crowley RW, Evans AJ, Kassell NF, et al. Endovascular treatment of a fusiform basilar artery aneurysm using multiple "in-stent stents." J Neurosurg Pediatr 2009;3:496-500 